\title{
Patients with congenital heart disease presenting for non-cardiac surgery
}

\author{
Kemp MEA \\ Department of Anaesthesia, Chris Hani Baragwanath Academic Hospital, University of the Witwatersrand \\ *Corresponding author, email: meakemp@mweb.co.za
}

\begin{abstract}
The survival rate of patients with congenital heart disease has increased markedly since 1980 , with over $80 \%$ of all patients now expected to reach adulthood. A growing population of survivors means that these patients will be presenting more commonly for anaesthesia and surgery for conditions unrelated to their heart. While some of these patients have good functional capacity, many will have residual symptoms such as arrhythmias, myocardial dysfunction and valvular lesions. A specific subgroup of patients who have only one functional ventricle and Fontan circulation is discussed with regards to the Fontan physiology and anaesthesia requirements.
\end{abstract}

Keywords: congenital heart disease, survival rates, mild, moderate, severe types, residual cardiac pathology, Fontan circulation, anaesthesia

\section{Introduction}

The number of patients surviving to adulthood with significant congenital heart disease is increasing and these patients may present for anaesthesia for conditions unrelated to their heart disease. Many of these patients will have significant residual cardiac morbidity or Fontan type physiology.

\section{Incidence of Congenital Heart Disease}

Congenital heart disease (CHD) is common, although the exact incidence is difficult to estimate accurately. Early estimates of $4-5 / 1000$ cases of live births (done in the 1950s) underestimated the true incidence. With better diagnostic modalities, the incidence of congenital heart disease is rising and now stands at 12-14/1000 live births. ${ }^{1}$ However, this incidence is also inaccurate because it does not take into account lesions such as bicuspid aortic valves (20/1000), hypertrophic cardiomyopathy (2/1000), or congenital arrhythmias (Wolff- Parkinson White, long QT) which only present in adolescents or adults, or asymptomatic abnormalities such as persistent left superior vena cava, tiny muscular ventricular septal defects or small patent ductus arteriosus that resolve in the first few years of life.

\section{Categories of Congenital Heart Disease}

It is useful to categorise CHD into severe, moderate and mild forms.

1. Severe congenital heart disease presents in the neonatal period or during infancy and often requires early cardiac surgery in a specialised unit.

Examples of these types of congenital heart disease include:

- Cyanotic heart disease and Tetralogy of Fallot

- d-Transposition of the Great Vessels (TGV)
- Hypoplastic right heart syndromes - such as tricuspid atresia, pulmonary atresia with an intact ventricular septum, Ebstein's anomaly

- Critical pulmonary stenosis or aortic stenosis

- Hypoplastic Left Heart syndromes with aortic hypoplasia, mitral atresia

- Truncus arteriosus

- Symptomatic co-arctation of the aorta

- Atrio-ventricular Canal defects (AVSD) or large Ventricular Septal Defects (VSD) or large Patent Ductus Arteriosus(PDA)

- Total Anomalous Pulmonary Venous Drainage (TAPVD)

2. Moderate $C H D$ is amenable to medical treatment until the child grows to a larger size, when corrective cardiac surgery or correction in the cardiac catherisation laboratory may or may not be indicated.

Lesions include:

- Moderate aortic stenosis or pulmonary stenosis or regurgitation

- Moderate mitral regurgitation or stenosis

- Non-critical aortic co-arctation

- Large Atrial Septal Defects (ASD)

- Moderate or complex VSD

3. Patients with mild congenital cardiac disease constitute the most numerous group and have disease that often presents with a murmur but with minimal or no symptoms.

- Small VSD, ASD, PDA

- Mild pulmonary stenosis

- Bicuspid aortic valve without regurgitation or stenosis 


\section{Prognosis of patients with congenital heart disease}

In 1940, only $30 \%$ of all patients diagnosed with congenital heart disease survived till adulthood; these patients had mild or moderate disease. The prognosis for severe disease was much worse, with only $5 \%$ of patients with more complex lesions surviving. The advent of cardiopulmonary bypass improved these statistics markedly - in the 1960 s $65 \%$ of patients with mild and moderate cardiac congenital heart disease could expect to survive until adulthood, and $15 \%$ of those with complex lesions would survive. From 1980 to the present times, there has been a marked increase in the survival of patients with congenital heart disease, with $85-90 \%$ of all patients with all lesions, mild, moderate and severe, now surviving to adulthood. In addition, whereas in the past, most patients with CHD who were seen by medical practitioners were children, now at least $50 \%$ of all patients with CHD are adults.

One of the questions asked is what is happening to these patients as they become older. Michael Silka's ${ }^{2}$ data from Oregon Health System looked at a cohort of 3500 patients. He found that most patients did well for the first ten to fifteen years, but after that, the rates of sudden death, cardiac failure and perioperative deaths are much greater than in the normal population.

Adults with CHD have increased cardiac morbidity and mortality due to the following factors ${ }^{3}$ :

\section{Arrhythmias:}

Many patients, particularly those with complex $\mathrm{CHD}$, will develop arrhythmias in their life time. Abnormal haemodynamic loading conditions, enlargement of the atria and multiple suture lines causing scarring in the heart predispose to atrial and ventricular arrhythmias. About $45 \%$ of patients with Fontan circulations will have atrial arrhythmias (which are usually difficult to control) and the incidence of ventricular arrhythmias in patients with corrected Tetralogy of Fallot and corrected Transposition of the Great Vessels is approximately $20 \%$ and $40 \%$ respectively. These arrhythmias predispose the patients to sudden death. At present, there is no satisfactory primary prevention for this complication.

\section{Heart Failure:}

Heart failure in all forms can be seen. This can be due to residual pulmonary hypertension or residual valvular lesions such as pulmonary regurgitation, causing right ventricular failure. About $10-15 \%$ of patients with treated left to right shunts (AVSD, VSD and ASD) will have pulmonary hypertension. Systolic failure of a single ventricle (commonly the right ventricle) occurs in univentricular hearts.

\section{Valvular lesions:}

Patients with repaired AVSD are prone to both mitral and tricuspid regurgitation and those with Tetralogy of Fallot commonly have pulmonary valve insufficiency and pulmonary regurgitation or pulmonary valve stenosis.

\section{Vascular lesions:}

Vascular lesions are common in this group - examples include re-stenosis of coarctation of the aorta; distortion, pre- or poststenotic aneurysmal dilation and re-stenosis of the branches of the pulmonary artery in patients with Fontan circulation and Tetralogy of Fallot.

\section{Residual shunts:}

Patients may have residual or new ventricular or atrial septal defects following surgery or may develop new aorto-pulmonary collaterals if they remain cyanosed.

\section{Fontan Physiology 4,5}

Patients with cardiac malformations that result in a single functional ventricle, such as tricuspid atresia, pulmonary atresia with an intact ventricular septum, double inlet ventricles or hypoplastic left heart syndrome, can now be treated by conversion to a Fontan type circulation.

\section{Establishment of a Fontan circulation}

The normal heart provides a double circuit circulation (pulmonary and systemic) that works in series and is powered by a double pump (right and left ventricle).

Many complex cardiac malformations are characterised by the presence of only one functional ventricle. If the patient is to survive during early post-natal life, the single ventricle must provide for both the systemic and pulmonary circulation. This can be by way of a natural connection between the pulmonary and systemic circulations, such as a patent ductus arteriosus or Major aorto-pulmonary collateral arteries (MAPCAs), or by an artificial connection such as a Blalock-Taussig shunt.

Such a parallel circulation circuit has many disadvantages; there is ongoing arterial desaturation, (with most oxygen saturations between $75-85 \%$ ), which becomes worse with exercise, as well as chronic volume overload on the single ventricle that causes gradual systolic heart failure. Few patients survive beyond the third decade of life with this type of circulation.

The solution, envisaged by Francis Fontan in 1971, was to separate the pulmonary from the systemic circulation by connecting the systemic venous return (via the superior and inferior vena cavae) directly to the pulmonary arteries (without the interposition of a pumping ventricle), thus leaving the single ventricle to supply the systemic arterial circulation. The advantages of this type of circulation are near normalisation of arterial oxygen saturation and abolishment of the chronic overload of the single ventricle.

In order for this to be successful, patients must have good myocardial function in the remaining ventricle, as well as low atrial pressures and a low trans-pulmonary gradient. The pulmonary vessels must be well developed; the pulmonary vascular resistance must be low; there must be unobstructed pulmonary venous return to the heart and there must be no resistance to the ventricular outflow tract. 
A Fontan circulation is established in stages; the first procedure, usually performed in the neonatal period, is the provision of a controlled supply of blood to the pulmonary artery from the ventricle. This provides a source of pulmonary blood flow for oxygenation and allows growth of the pulmonary vasculature. It is important to prevent excessive pulmonary blood flow with resulting pulmonary artery hypertension and increased pulmonary vascular resistance.

This is usually done via a modified Blalock-Taussig (BT) shunt, (which uses a graft to supply blood from the right subclavian artery to the right pulmonary artery), or a Sano shunt (a graft directly from the ventricle to the pulmonary artery), or, in the case of excessive blood flow to the lungs, (left heart hypoplasia) pulmonary artery banding. In the case of left heart hypoplasia, a Norwood procedure to correct the hypoplasia of the aorta is also performed as unrestricted blood flow from the ventricle to the aorta is required.

After allowing for adequate growth of the pulmonary vessels, and after careful evaluation of the pulmonary vasculature, the first stage of the Fontan is then performed. This is often within the first year of life. The most popular procedure used currently to connect the superior vena cava to the pulmonary artery is called the bi-directional Glenn shunt. Any aorto-pulmonary shunts (such as the BT shunt) are taken down so that the lungs are supplied directly from the superior vena cava alone. A fenestration is often placed within the atrium to provide a 'pop - off' from the superior vena cava if the pulmonary vascular resistance should rise.

After this stage, the patient continues to have arterial desaturation, because the inferior vena cava still returns to the heart and there is ongoing volume overload of the ventricle.

If the bi-directional Glenn shunt is well tolerated, most patients should be converted to a total cavo-pulmonary shunt within 18 months (preferably before the age of 5 years), in order to prevent deterioration of ventricular function due to chronic overload.

To complete the Fontan circulation, the inferior vena cava is anastomosed to the pulmonary artery. This may be done in two ways. The lateral tunnel method provides a tubular pathway between the inferior vena cava and the pulmonary artery using a prosthetic baffle and a portion of the lateral atrial wall. This is useful because it has potential for growth and so can be performed in a young child. However, it does leave the atrial tissue exposed to relatively high venous pressures, which in turn predisposes to atrial arrhythmias.

The extra-cardiac conduit consists of a tube graft between the inferior vena cava and the pulmonary artery. This can be placed without cardiac bypass but has no potential for growth and therefore can only be performed in older patients. Completion of the Fontan allows for arterial saturation to become low normal ( $90-95 \%)$ and offloads the ventricle.

Mortality assessment in patients with Fontan circulation is always considerably biased - bias occurs firstly when the patient is initially accepted for a Fontan procedure and then if they survive the procedure. Many patients with Fontan circulation survive for several decades with reasonable haemodynamic function but those patients who have unfavourable haemodynamic function have increased early and late mortality.

\section{Haemodynamic consequences of a Fontan circulation ${ }^{6}$}

Increased systemic venous pressure is necessary in a Fontan circulation because of the requirement for a driving pressure from the systemic venous system, via the cavo-pulmonary connection, to the pulmonary vascular bed. There is a complicated and dynamic relationship between the resistance characteristics in this circuit. As resistance to venous return increases, (due to increases in cavo-pulmonary and pulmonary vascular resistance) the preload to the pumping ventricle decreases.

The increased systemic venous pressure means that patients with Fontan circulations exhibit tolerance to orthostatic hypotension but are very vulnerable to acute decreases in intra-vascular volume and vasodilation.

The cardiac output in a Fontan circulation depends directly on the passive flow of blood through the lungs to supply the preload to the ventricle. Fontan circulations inherently have a decreased ventricular preload due to the non-pulsatile, low pressure pulmonary driving force, as well as an increased afterload as a result of the arrangement of three resistance vascular beds in series (systemic, cavo-pulmonary and pulmonary). Therefore, cardiac output is limited.

Despite this, more than $90 \%$ of Fontan survivors are in New York Heart Association functional class 1 or 2.

\section{Problems associated with Fontan type physiology}

1. High venous systemic pressures

The increased systemic venous and caval pressures are transmitted to the liver, resulting in chronic hepatic venous congestion, which, together with the low systemic cardiac output, results in hepatic inflammation, fibrosis and cirrhosis called FALD (Fontan Associated Liver Disease). The ubiquitous presence and insidious onset of liver disease in Fontan patients probably justify routine screening.

\section{Lymphatic dysfunction}

A Fontan circulation operates at the functional limits of the lymphatic system because of the high venous pressures and with run-offintothesuperiorvenacavaimpeded.Peripheraloedemacan occur and leakage of lymphatics into the lungs causes pulmonary oedema and plastic bronchitis, which has a poor prognosis. Leakage into the gut results in Protein Losing Enteropathy (PLE), which causes malabsorption, malnutrition and immune deficiency.

\section{Coagulopathy}

Thrombosis is more likely to occur in patients with a low cardiac output, atrial dilation and systemic stasis. A systemic venous thrombosis has the potential to embolise to the pulmonary circulation and is the most common cause of out-of-hospital death in Fontan patients. Some clinicians recommend anticoagulating every patient with a Fontan circulation; however, 
there are subgroups of patients who are at low risk. It is not clear if low dose aspirin or clopidogrel is beneficial to these patients, but these patients are often placed on these agents.

\section{Ventricular function}

All studies of univentricular hearts show them to be dilated, hypertrophic and hypocontractile.

Patients with Fontan circulations performed under ideal circumstances (i.e. staged procedure completed before the age of 5 years) have a lower than average stroke volume (although most have an ejection fraction within 2 standard deviations of normal) due to the decreased preload and increased afterload. The ventricle prior to completion of the Fontan is always volume overloaded and after completion, the preload is reduced to between $50-70 \%$ of normal - thus the ventricle evolves from being volume overloaded and overstretched to overgrown and underloaded. Remodelling caused by chronic underfilling can cause a declining cardiac output. The type of ventricle present also plays a part - a left ventricle has a shape and muscle configuration that is suitable to sustain a lifetime of systemic pressure pumping, but an undifferentiated ventricle or a right ventricle is not.

The treatment of a failing ventricle in a Fontan circulation is frustrating as many studies show that conventional medication, such as beta-blockers, vasodilators and inotropes have little effect (which is not surprising, given that reduced preload is the problem).

\section{Arrythymias}

Patients with a Fontan circulation are predisposed to developing atrial arrhythmias, particularly if part of the atrial wall is included in the cavo-pulmonary anastomosis. Up to $40 \%$ of patients who have had a Fontan for 10 years will develop atrial reentrant tachyarrhythmias. These lead to rapid haemodynamic compromise and usually require treatment with DC shock conversion. Long term treatment includes drugs (noting that these patients are extremely sensitive to decreased contractility and changes in vascular resistance) and ablation in the ElectroPhysiology laboratory. The best long-term treatment appears to be conversion to an extra-cardiac Fontan, with or without an epicardial pacemaker. Ventricular arrhythmias are rare, except in the context of poor haemodynamic function.

\section{Hypoxaemia caused by residual shunts}

Normal oxygen saturation for Fontan patients is in the low $90 \%$ s. Patients may have residual fenestrations between the atrial wall and the cavo-pulmonary connection or other connections between the pulmonary and systemic circulations (such as drainage of the coronary sinus into the systemic atrium), which can cause symptomatic cyanosis.

Some patients have residual aorto-pulmonary collaterals or are predisposed to developing such collaterals. These collaterals usually place an unimportant volume load on the systemic ventricle but can increase pulmonary vascular resistance and impede venous blood flow through the lungs.

The effect of high altitude and decreased oxygen tensions varies widely amongst individuals although it is recommended that patients with borderline haemodynamic function should not live above 2000 metres above sea level.

\section{Anaesthesia and patients with Fontan Circulation?}

\section{Preoperative considerations:}

It is important to evaluate the functional capacity of patients with Fontan circulations pre-operatively. Patients with good exercise tolerance do well with anaesthesia. In the long term however, about $70 \%$ of patients with Fontan physiology have some myocardial dysfunction and about $45 \%$ develop atrial arrhythmias.

Liver function should also be investigated, given the high incidence of Fontan associated liver disease.

Peripheral oedema and malnutrition can occur as a result of protein losing enteropathy.

Some patients are at increased risk of thromboembolism as a result of low flow states. Aspirin should probably be discontinued if there is sufficient time to do so (one week). Bridging therapy with low molecular weight heparin should be given in individual cases who are at high risk of thrombosis (low output states, prior thrombosis, hypercoagulable states).

\section{Intraoperative considerations:}

There is a risk of systemic fat or air embolism if the patient has a fenestration in the atrium. Great care should therefore be taken to thoroughly de-air intra-venous lines and to avoid injecting air together with intra-venous boluses.

Intra-arterial blood pressure monitoring should be used in moderate and major surgical cases as patients are very sensitive to changes in systemic vascular tone and fluid volume.

Be cautious when inserting central lines - any line placed in the internal jugular, subclavian or femoral veins is in direct contact with the pulmonary artery and therefore is actually a pulmonary arterial catheter. Adequate pulmonary blood flow and systemic cardiac output depends on unobstructed blood flow through the cavo-pulmonary connection and if these are damaged or obstructed, significant haemodynamic consequences can ensue. Monitoring of the central venous pressure reflects mean pulmonary arterial pressure - typically, in a well Fontan patient, this is about $12-15 \mathrm{mmHg}$.

Infective endocarditis prophylaxis should always be given to these patients; they generally have vascular grafts present in the circulation and commonly have atrioventricular valve dysfunction.

\section{Haemodynamic goals:}

The main determinants of a good cardiac output in a Fontan circulation are:

Increased venous pressure - the intra-vascular volume is one of the main determinants of pulmonary blood flow, so that hypovolaemia and vasodilation are generally poorly tolerated. Dehydration and blood volume loss should be replaced timeously. Maintain venous tone, particularly in the lower limbs 
and splanchnic bed, as the inferior vena cava supplies about $65 \%$ of the venous return to the lungs.

Low pulmonary vascular resistance - care should be taken to avoid factors that increase this such as alveolar hypoxia, hypercarbia, acidosis, hyperthermia and drugs such as a adrenergic agents.

An unobstructed cavo-pulmonary connection - this is an anatomical factor that is generally beyond control.

\section{Ventricular function}

Many patients will have some myocardial dysfunction as a result of chronic underloading of the ventricle or residual atrioventricular valve regurgitation. Induction and maintenance of anaesthesia should avoid agents with myocardial depressant effects. Use of vasoconstrictors may be required to address venous vasodilation but as all current agents are also arterial vasoconstrictors, the resultant increase in afterload may compromise ventricular function.

\section{Ventilation in patients with Fontan physiology:}

One of the side effects related to the inevitable increase in the mean intra-thoracic pressure that accompanies positive pressure ventilation is a decrease in venous return, with a concomitant decrease in pulmonary blood flow and decreased cardiac output.

The effects of positive pressure ventilation on pulmonary blood flow are of concern in Fontan physiology, although the effects have not been investigated systematically. It has been suggested that there is a near linear relationship between mean airway pressure and cardiac output in Fontan patients.

Doppler studies in normal patients show that pulmonary blood flow is $35 \%$ greater during inspiration than expiration and $30 \%$ greater in Fontan patients. In Fontan patients, the effect on cardiac output of the respiratory component of venous return to the heart is $30 \%$ as compared to $15 \%$ in two ventricle physiology. (Note that the absence of two ventricles also eliminates the possibility of pulsus paradoxus.)

While spontaneous ventilation in Fontan patients is associated with a higher cardiac output as compared to during positive pressure ventilation, the presence of hypoxaemia, hypercarbia and low lung volumes associated with inadequate spontaneous ventilation increases pulmonary vascular resistance and this may well cause a more significant drop in cardiac output than well managed controlled positive pressure ventilation.

Many Fontan patients cope well with positive pressure ventilation, if the mean airway pressure is kept low (less than $15-20 \mathrm{mmHg}$ ), if Positive End Expiratory Pressures (PEEP) is kept low and pulmonary vascular resistance is kept low with high inspired oxygen concentrations, low-normal arterial carbon dioxide levels, and avoidance of excessive adrenergic stimulation (pain, drugs).

\section{Fontan patients and laparoscopic surgery:}

There have been case reports of successful laparoscopic surgery performed in Fontan patients, particularly laparoscopic appendectomies and the procedure is well tolerated in Fontan patients with good functional capacity. Most reports recommend maintaining intra-abdominal pressures below $10 \mathrm{mmHg}$ and warn about the risk of paradoxical $\mathrm{CO}_{2}$ embolism in patients with fenestrations. Adequate hydration and ventilation and minimal surgical time are also recommended.

\section{Pregnancy:}

Most women with Fontan circulation have normal menstrual patterns and women with good haemodynamic function tolerate pregnancy and labour well. Assisted vaginal delivery is recommended.

\section{Cardio-pulmonary resuscitation (CPR) in a patient with a Fontan circulation:}

Conventional CPR has limited efficacy in patients with Fontan circulation. During chest compressions, increased atrial pressure may impede blood returning to the heart from the lungs, reducing preload and further compromising cardiac output. The situation is worsened because the patients also often have atrioventricular valve regurgitation.

There is no compressible chamber or valve between the superior and inferior vena cava and the pulmonary artery, rendering the compression-release mechanism of CPR less effective (i.e. the blood tends to go back into the head and abdomen rather than forwards into the lungs). The acidosis and hypercarbia associated with the arrest elevate pulmonary vascular resistance, further impeding forward flow into the lungs. There are case reports of abdominal compressions used together with chest compressions that resulted in increased arterial pressure, but in the presence of hepatic congestion, this may well not be advisable.

\section{Conclusion}

As a result of the markedly improved survival of patients with mild, moderate and severe congenital heart disease, there is a growing population of children and adults who will present for emergency and elective anaesthesia and surgery unrelated to their cardiac condition. Patients with good functional capacity will usually cope well with the physiological demands of surgery and anaesthesia although a significant number of survivors with congenital heart disease will have significant cardiac morbidity by way of arrhythmias and myocardial dysfunction. A specific subset of patients to be aware of are those who have a single ventricle and Fontan circulation.

\section{References:}

1. Daniels CJ. Current Trends in the Prevalence of Adults with Congenital Heart Disease (Slides with Transcript). Available from: https://doi.org/10.1161/01. CIR.92.4.758

2. Maron BJ, Gardin JM, Flack JM, Giiding SS, Kurosaki TT, Bild E. Circulation.1995;92:785-9.

3. Ministeri M, Alonso-Gonzalez R, Swan L, Dimopoulos K. Common Long Term Complications of Adult Congenital Heart Disease: Avoid Falling in a H.E.A.P. Expert Rev Cardiovasc Ther. 2016 [Epub 25 Jan 2016];14(4):445-62. doi:10.1586/ 14779072.2016.1133294

4. Gewillig M. The Fontan Circulation. Heart. Jun 2005:91(6):839-46.

5. Nyak S. The Fontan Circulation. Continuing Education in Anaesthesia Critical Care and Pain. February 2008;8(1):26-30. Available from: https://doi.org/10.1093/ bjaceaccp/mkm047

6. Jolley M, Colan SD, Rhodes J, DiNardo J. Fontan Physiology Revisited. Anaesth Analg. July 2015;121(1):172-82. Available from: https://doi.org/10.1213/ AME.0000000000000717

7. Harikrishan K, Lim ML, Shah SKS. Fontan Physiology: Implications for Non-Cardiac Surgery: A Case Report. Int J. Anesth Anesth. 2015;2:020. Available from: htttps://doi.org/10.23937/2377-4630/2/1/1020 